\title{
Egreso universitario a término: elementos que permiten comprender su trama
}

\author{
Graduation from College on Time: Elements that Allow to \\ Understanding its Plot
}

Natalia Soledad Petric ${ }^{1}$

Resumen: Se aborda el tema del egreso a término en carreras universitarias que representan cinco ramas disciplinares (ciencias sociales, humanas, aplicadas, básicas y de la salud), desde la voz de los egresados. El objetivo fue desentrañar las dimensiones implicadas, además de construir categorías teóricas sustantivas que den cuenta de las experiencias de dichos actores universitarios.

Se utilizó un diseño cualitativo de investigación a partir de entrevistas focalizadas a treinta y tres egresados de carreras de grado de universidades de gestión estatal de la región centro argentina. El análisis se realizó desde la perspectiva de la teoría fundamentada en la versión de Strauss y Corbin (2002).

Se arribó a la idea de trama, que permite describir y explicar el modo complejo de interrelación entre las categorías centrales o puntos de la trama. Estas categorías fueron equipaje, autopercepción estudiosa, estrategias de avance y una categoría contextual: modalidad de cursado de la carrera; todas ellas con sus correspondientes propiedades y dimensiones.

Palabras clave: grado universitario, alumno egresado, investigación cualitativa.

\footnotetext{
${ }^{1}$ Doctora en Psicopedagogía. Licenciada en Psicopedagogía. Profesora de Educación Especial. Docente e investigadora de la Universidad Católica Argentina. Docente de la Universidad de la Cuenca del Plata. Integrante del Programa de ingreso, permanencia y egreso universitario de la Universidad Autónoma de Entre Ríos. Paraná, Entre Ríos, Argentina. Correo electrónico: nataliapetric@hotmail.com.
}

Diálagos Pedagágicas. ISSN en línea: 2524-9274.

Año XVII, No 34, octubre 2019 - marzo 2020. Pág. 61-82.

DOI: http://dx.doi.org/10.22529/dp.2019.17(34)04 / Recibido: 9-08-2018 / Aprobado: 10-05-2019.

Artículo publicado bajo Licencia Creative Commons Atribución-NoComercial-SinDerivar. (C) Universidad Católica de Córdoba. 
Abstract: The issue of graduating on time from college careers representing five disciplinary branches (social, human, applied, basic, and health sciences) is approached from the opinion of graduates. The objective was to unravel the involved dimensions, as well as to construct substantive theoretical categories that give account of the experiences of said university actors.

A qualitative research design was used, based on interviews to 33 graduates of degree programs from state-run universities in the Central Region of Argentina. The analysis was made from the perspective of the theory based in the version of Strauss \& Corbin (2002).

We arrived at the idea of a plot, which allows us to describe and explain the complex interrelationship between the focus categories or points of the plot. These categories were baggage, studious self-perception, advance strategies, and a contextual category: modality of career course, all of them with their corresponding properties and dimensions.

Keywords: academic degree, graduate student, qualitative research.

\section{Introducción}

El presente artículo expone los principales resultados de una investigación acerca del egreso a término en la universidad ${ }^{2}$ y se inscribe en la problemática de los estudios universitarios de grado (pre-grado en algunos países).

La cuestión del tránsito por la universidad ha sido ampliamente descripta y analizada desde diferentes perspectivas. Esto evidencia la centralidad de la temática en las instituciones de educación superior. No obstante la extensión de su tratamiento, frecuentemente, es abordada desde sus manifestaciones negativas como lo son el abandono de la carrera, la prolongación de los tiempos de cursado, las estrategias inadecuadas de estudio, sobre todo en los primeros años, y la desarticulación entre el nivel medio y el superior.

Es menos habitual encontrar estudios referidos al egreso en el nivel universitario, es decir, destinados a conocer a los estudiantes que obtienen resultados satisfactorios, que avanzan en la carrera y finalizan los estudios.

Los relativamente escasos egresados que tiene el sistema, en relación con los ingresos crecientes de las últimas décadas (Barsky, Sigal \& Dávila, 2004; Ezcurra, 2013, 2014; García de Fanelli, 2014a, 2015; Rama, 2007), parecen indicar que, para algunos sectores, egresar de la universidad con un título de grado es la excepción.

2 El artículo se desprende de los hallazgos producto de la investigación, que fue el sustento de la tesis de Doctorado en Psicopedagogía La trama del egreso a término. Un estudio en egresados de universidades de gestión estatal de la Región Centro argentina. Fue rendida y aprobada con nota 10 (diez) en 2017. Facultad de Psicología y Psicopedagogía, Pontificia Universidad Católica Argentina. 
Es así que el egreso se ha convertido en una preocupación a nivel mundial a partir de considerar lo que podría ser su sombra: la deserción. En Estados Unidos, esta ronda el $47 \%$, igual que Suecia y Hungría. En Nueva Zelanda y Noruega, es algo más baja: $41 \%$. En Reino Unido, Holanda, República Checa, Eslovaquia, Portugal, México y Polonia, oscila entre $28 \%$ y $38 \%$, mientras que, en Japón y Dinamarca, es menor al 20\% (Ezcurra, 2014).

Por esto, cuando las cifras de los estudiantes que abandonan las instituciones educativas sin obtener el título son elevadas, como en el caso de los universitarios, surge la pregunta acerca de quiénes "sobreviven en el sistema". ¿Por qué algunos estudiantes pueden persistir en las mismas instituciones donde tantos no continúan o demoran largamente su egreso? Y, más específicamente, ¿qué dimensiones se ponen en juego para egresar en el tiempo que estipula el plan de estudios?

En este sentido y desde la lógica cualitativa, se indagaron de manera central las dimensiones implicadas en el egreso universitario a término, tal como los actores (egresados) construyeron el relato y las significaciones en torno al egreso, de modo de comprender, desde una perspectiva microsocial, las profundas y heterogéneas experiencias y las configuraciones que adquirieron las dimensiones intervinientes. Si bien la investigación que originó este artículo abordó también aspectos vinculados a las configuraciones disciplinares emergentes en cada carrera estudiada e indagó los significados construidos en torno al aprendizaje universitario, aquí solo se presentarán los hallazgos acerca de la trama construida entre las categorías centrales (equipaje, autopercepción estudiosa, modalidad de cursado y estrategias para avanzar) y sus propiedades respecto al egreso a término.

\section{Contextualización del problema}

La preocupación por los estudios universitarios en Argentina se correlaciona con la expansión de la matrícula de la educación superior a lo largo de todo el siglo XX y comienzos del XXI con una tasa de crecimiento promedio del siete por ciento anual (García de Fanelli, 2005). Esto conlleva una de las tasas brutas de escolarización superior más altas de Latinoamérica.

La Secretaría de Políticas Universitarias de la Nación (SPU), en su Anuario de Estadísticas correspondiente al año 2006 (Argentina. Ministerio de Educación de la Nación, 2006), informa que Argentina presenta la tasa bruta de escolaridad de nivel superior más alta de América Latina. Está seguida por Panamá, Chile, Costa Rica, Venezuela; Honduras y El Salvador se ubican en los últimos lugares.

Si bien la expansión cuantitativa de la enseñanza superior se muestra a través de tasas de cobertura optimistas, los sistemas educativos, incluido el argentino, enfrentan desafíos importantes en relación al cumplimiento de las metas y objetivos formulados desde las políticas educativas. Los bajos rendimientos se manifiestan de distintas formas preocupantes: la diferencia que existe entre los tiempos teóricos y los tiempos reales de duración de una carrera, el 
desgranamiento, el promedio de egresados en las instituciones, el abandono de las carreras universitarias, etc.

En las últimas dos décadas, la mejora en los índices de rendimiento académico y egreso se ha incorporado como tema de alta relevancia en la agenda de políticas públicas e institucionales en América Latina (Brunner \& Ferrada Hurtado, 2011).

Según un informe del Instituto Internacional para la Educación Superior en América Latina y el Caribe (IESALC), la eficiencia de titulación (medida por el número de los que se gradúan dentro de los cinco años previstos) alcanza, en América Latina, un promedio del 43,2\%. Argentina se encuentra cerca del $11 \%$; Brasil estaría en un promedio del $26 \%$ para universidades públicas y del $46 \%$ para universidades privadas; Chile, $50 \%$ para universidades públicas y $37,4 \%$ para universidades privadas y Cuba, 75\% (Pérez Lindo, 2007).

La duración efectiva de las carreras, entonces, promedia entre siete u ocho años. A su vez, esto configura una situación de atraso escolar para la mayoría de los estudiantes que accede a su primer diploma cerca de los 30 años. Estos estudiantes quedan limitados o bloqueados para culminar estudios de posgrado.

En Argentina, la preocupación por los altos índices de deserción y bajos índices de egreso toma relevancia a partir de la década del '90. La Secretaría de Políticas Universitarias (SPU) incorpora, en su Anuario de Estadísticas Universitarias (Argentina. Ministerio de Educación de la Nación, 2005), una estimación de la eficiencia de titulación al considerar el cociente entre los nuevos inscriptos y los egresados seis años después. Y expresa que, en promedio, de cada 100 inscriptos en 20 carreras seleccionadas en universidades nacionales, solo 19 egresaban aproximadamente- dentro del plazo establecido por los planes de estudio (García Fanelli, 2014a, 2015).

Asimismo, es interesante mencionar un clásico estudio realizado en Argentina por Landi y Giuliodori (2001), quienes señalan que el problema del egreso y la deserción se entrecruza con el de la duración de los estudios. Los autores basan su investigación en la EPH (Encuesta Permanente de Hogares) aplicada por el Instituto Nacional de Estadística y Censos (INDEC) en 1998. Se aprecia que el $99 \%$ de los egresados comprendidos en la muestra declaró haber completado la carrera en el término de 15,7 o menos años; vale decir, dentro del decimosexto año académico a partir del comienzo de sus estudios.

Además de señalar la dificultad para establecer tasas de egreso y deserción, el mismo informe comenta que, en Argentina, no hay estudios longitudinales sistemáticos que muestren el comportamiento de las cohortes para establecer las tasas de deserción, egreso y duración real de los estudios. Por lo tanto, hay estimaciones efectuadas por procedimientos diversos y de difícil comparación.

García de Fanelli (2014b) expresa la manera en que el aumento en la tasa de egreso y la reducción del tiempo para "formar un egresado" se constituyen en índices de calidad y eficiencia organizacional, y refiere que, en Argentina, la baja tasa de egreso universitario es un problema de vieja data. Vinculado a esto, un censo de la Universidad de Buenos Aires de 1958 habría alertado acerca de la 
dilación en la duración de las carreras en un $60 \%$ más de lo que proponían los planes de estudios (Germani, 1965, citado por Carli, 2012).

Es así que muchas de las producciones estadísticas que sondean los fenómenos universitarios y circunscriben su objeto a la finalización de las carreras en el tiempo que propone el plan de estudios utilizan el término eficiencia terminal. Este término es vinculado con la calidad de la educación superior.

Internacionalmente, la eficiencia terminal (educational efficiency) es definida por la Unesco como el grado en el cual un sistema educativo consigue optimizar la relación inversión-resultado en educación (López Suárez, Albíter Rodríguez \& Ramírez Revueltas, 2008).

Pérez Gonzáles (2006) plantea la eficiencia terminal desde tres posibles maneras de estudiarla: cociente del número de alumnos que concluyeron la totalidad de sus estudios en un año determinado entre los alumnos que ingresaron en el mismo año; cociente del número de alumnos que egresan o se titulan en un año dado sobre el ingreso en un periodo de años anterior según el tiempo de duración de la carrera y seguimiento del desempeño individual hasta el egreso y titulación.

Con relación a su utilidad, la eficiencia terminal es descripta como un índice cuantitativo, una "medida resumen" (Camarena, Chávez \& Gómez, 1985) de la trayectoria estudiantil, ya que se posiciona entre los polos del ingreso y del egreso. Es indicador tanto de la eficiencia como de la calidad del sistema y de su rendimiento; un "termómetro" de la vida académica, de los programas de estudio y de los egresados (Edel Navarro, 2004).

A su vez, se plantean dificultades en torno a su definición. Las principales remiten a la dificultad en su medición, dados los desacuerdos relativos al significado de los términos ingreso y egreso, especialmente cuando no existe información precisa sobre estos momentos. (Cuéllar Saavedra \& Bolívar Espinoza, 2006).

Si no existen los datos, se recurre a estimaciones indirectas: la más frecuentemente usada en educación superior es la que relaciona el número de titulados (o egresados), en un momento determinado (un año dado), con el total de los que iniciaron un cierto número de años antes (normalmente seis años).

Como corolario, las bajas tasas de egreso, y más aun de eficiencia terminal, invitan a reflexionar acerca de una problemática que afecta tanto a los sujetos como a las instituciones, y por ende, a la sociedad en su conjunto, a pesar de que se han observado aumentos en las tasas a nivel iberoamericano.

Asimismo, se resalta la necesidad de generar estudios en los que se devele la perspectiva de los sujetos que han transitado la experiencia de egresar a término en sus carreras desde propuestas microsociales que permiten pensar el egreso a término como constructo que remite a un proceso que tiene un inicio y un fin, ambos prediseñados por las políticas educativas a través de los planes de estudio, aunque transitados de diversas maneras por los sujetos. Es necesario focalizar en los procesos y dimensiones que se ponen en juego en esos trayectos y no en los índices que miden estadísticamente magnitudes. 


\section{Metodología}

El estudio acerca del egreso universitario -ya sea a término o no- ha sido principalmente abordado desde perspectivas cuantitativas, (Cabrera, Tomás, Álvarez \& González, 2006; Castaño, Gallón, Gómez \& Vásquez, 2006; García de Fanelli, 2005; Landi \& Giulodori, 2001; Oddino, 2003; Sánchez, Quirós, Reverón \& Rodríguez, 2002).

Por este motivo, se optó por un diseño cualitativo que permitiera dar cuenta de las experiencias de los sujetos y las maneras en que construyen el egreso a término desde la perspectiva de los egresados, "sus percepciones, ideas, emociones e interpretaciones" (Sautu, 2005, p. 71). Y, de manera inductiva, se elaboró una teoría sustantiva respecto al fenómeno. Es por esto que se realizaron entrevistas en profundidad a treinta y tres graduados, se entrevistó a informantes clave y se analizaron documentos.

Se seleccionaron cinco carreras, que representaron las ramas disciplinares propuestas por el Ministerio de Educación argentino en sus Anuarios Estadísticos y por instituciones universitarias de la Región Centro argentina. Es así que se trabajó con las siguientes carreras: Profesorado en Ciencias de la Educación (ciencias humanas), Ingeniería Química (ciencias aplicadas), Abogacía (ciencias sociales), Licenciatura en Física (ciencias básicas) y Licenciatura en Nutrición (ciencias de la salud).

La perspectiva metodológica utilizada para el análisis de las entrevistas a los egresados fue la Teoría Enraizada o Grounded Theory, desde la reformulación de Strauss y Corbin (2002), y se utilizó el programa de análisis cualitativo Atlas Ti 6.2 para el procesamiento de los datos.

\section{Hallazgos}

Las categorías construidas permitieron la emergencia de la metáfora de la trama como tejido que muestra un entrelazado de categorías que dan cuenta de la complejidad del fenómeno. Hablar de trama remite al paradigma de la complejidad (Morin, 2009). Esta complejidad, lejos de aludir a lo enmarañado, complicado o difícil de entender y atender, permite ampliar la mirada y pone en relación múltiples hilos, como un conjunto amplio y complejo de relaciones.

Las categorías centrales o puntos en la trama (en referencia al sentido que adquiere el tejido) permitieron dar cuenta de la trama común del egreso a término en las cinco carreras estudiadas. En esta construcción, se utilizó un criterio de maximización de similitudes propuesta por la teoría fundamentada (Glaser \& Strauss, 1967).

Estas categorías se denominaron equipaje, autopercepción estudiosa y estrategias para avanzar. Las dos primeras presentan escasa variación según las diferentes carreras estudiadas y la tercera se configura según la modalidad de cursado de la carrera estudiada (cursado estructurado o cursado no estructurado). 
La modalidad de cursado de cada una de las carreras estudiadas se definió como el contexto o el conjunto de condiciones intervinientes que influencian las estrategias (Strauss \& Corbin, 2002) y no como una categoría emergente de los datos.

Se resalta la metáfora de la trama como hipótesis que permitió interpretar los modos en que se relacionaron estas categorías.

\section{Equipaje}

Esta categoría se construyó con base en un código in vivo proveniente de la entrevista a Norma, egresada del Profesorado en Ciencias de la Educación, y refiere al conjunto de experiencias pasadas y actuales que conforman una actitud favorable hacia el estudio. Incluye -principalmente- aspectos familiares, aunque -también- referencias a experiencias educativas previas.

Generalmente, estas experiencias expresan un contínuum desde vivencias previas, tanto de educación escolar como familiar, hasta la universidad. Así lo expresó Norma:

"Es como que yo estaba equipada con un par de cositas que me ayudaron a afrontar ese cambio [con relación al pasaje escuela secundaria- universidad]. Entonces, en ese sentido, yo creo que, como me gustaba, me daban las ganas de leer, de querer saber, todo me interesaba, todo era nuevo". (Norma, egresada de Profesorado en Ciencias de la Educación)

La Tabla 1 da cuenta de la categoría, sus propiedades y dimensiones.

Tabla 1. Propiedades y dimensiones de la categoría equipaje

\begin{tabular}{|c|c|c|c|}
\hline Categoría & Propiedad & Dimensión & Consecuencia \\
\hline \multirow[t]{5}{*}{ Equipaje } & $\begin{array}{l}\text { Mandato familiar de } \\
\text { estudiar. }\end{array}$ & Implícito - explícito. & \multirow{4}{*}{$\begin{array}{l}\text { No trabajar } \\
\text { Esfuerzo por } \\
\text { sacrificio }\end{array}$} \\
\hline & $\begin{array}{l}\text { Ser -o no- la primera } \\
\text { generación de estudiantes } \\
\text { universitarios. }\end{array}$ & $\begin{array}{l}\text { Estudios primarios } \\
\text { (completos-incompletos) } \\
\text { a posgrados } \\
\text { (completos-incompletos). }\end{array}$ & \\
\hline & Monitoreo familiar. & $\begin{array}{l}\text { De más exigente a más } \\
\text { acompañado. }\end{array}$ & \\
\hline & $\begin{array}{l}\text { Apoyo económico } \\
\text { familiar. }\end{array}$ & $\begin{array}{l}\text { Trabajo para sostenerse } \\
\text { económicamente - trabajo } \\
\text { por elección. }\end{array}$ & \\
\hline & $\begin{array}{l}\text { Buenas experiencias } \\
\text { educativas previas. }\end{array}$ & $\begin{array}{l}\text { Desde una mirada crítica } \\
\text { o negativa a una valora- } \\
\text { ción positiva. }\end{array}$ & \\
\hline
\end{tabular}


A modo ilustrativo, se exponen textualidades que remiten al monitoreo familiar. Esta propiedad refiere al grado de acompañamiento y supervisión familiar respecto de actividades vinculadas al estudio. Dimensionalmente, va desde un monitoreo más exigente y de control a uno más acompañado y de apoyo. A su vez, esta supervisión representó un contínuum desde experiencias educativas previas y continuó durante la universidad.

En este sentido, Norma expresó el lugar del acompañamiento materno en todo lo referido al ámbito escolar:

"Desde la primaria incluso, el hecho de sentarse a hacer la tarea con nosotros, estar siempre muy pendiente, acompañarnos, llevarnos, traernos, buscarnos, si necesitábamos algo. O sea, siempre muy ocupados y como que la escolaridad de todas fue LO importante [...]. No hicimos actividades extraescolares, quizás no fuimos a inglés, nos fuimos a danzas, como por ahí han hecho otros chicos. Pero la escuela siempre era como lo central". (Norma, egresada del Profesorado en Ciencias de la Educación)

$\mathrm{Y}$, desde un polo más exigente, vinculado al conocimiento de los ritmos universitarios, en este caso, los turnos de exámenes:

"Otro factor que te puedo mencionar es mi mamá que fue muy exigente, que todos los turnos me presente algo [...]. Pero con mi mamá me pasaba una cosa más de no sé cómo decirte... De tratar de que ella no se enoje porque yo no rendía. Por el reconocimiento, a lo mejor. Y era como una decepción para ella si yo no me presentaba [...] como una presión: '¿Qué vas a rendir este turno?, ¿y en este no vas a rendir nada?'". (Melisa, egresada de Abogacía)

\section{Autopercepción estudiosa}

La siguiente categoría remite a cuestiones vinculadas a las definiciones que realizaron los entrevistados respecto de sí mismos con relación al estudio. En la mayoría de los casos, las propiedades que la conformaron -ser aplicado; ser autoexigente; ser tenaz- se manifestaron en los relatos desde un contínuum con experiencias previas y se relacionaron, de manera directa, con la categoría equipaje.

La Tabla 2 da cuenta de la conformación de esta categoría central.

Tabla 2. Propiedades de la categoría autopercepción estudiosa

\begin{tabular}{|l|l|l|}
\hline Categoría & \multicolumn{1}{|c|}{ Propiedad } & \multicolumn{1}{c|}{ Dimensión } \\
\hline \multirow{2}{*}{$\begin{array}{l}\text { Autopercepción } \\
\text { estudiosa }\end{array}$} & Ser aplicado & $\begin{array}{l}\text { Todas las propiedades pudieron } \\
\text { encontrarse de manera más o } \\
\text { menos acentuada. }\end{array}$ \\
\cline { 2 - 3 } & Ser autoexigente & \\
\cline { 2 - 3 } & Ser tenaz & \\
\hline
\end{tabular}


Para ilustrar esta categoría, se presentan extractos de los relatos de los entrevistados que señalan cómo el gusto por el estudio se manifestó ligado al sentido de responsabilidad y a la disciplina:

"Soy re responsable mal. Si empiezo algo, lo quiero terminar. Y bueno, la disciplina. Y no me cuesta tampoco estudiar. O sea que me gusta. [...] Estructurada a fondo. Y sí, no te queda otra [...] Todo el tiempo libre que yo tenía, lo aprovechaba". (Victoria, egresada de Abogacía)

Además de considerar el estudio como un trabajo, lo que les significó tener autodisciplina para sostener los momentos de desgano:

"Yo me tomaba la universidad como una especie de trabajo. Si no había ganas, me obligaba a tratar de mantener el ritmo, a tratar de hacer las cosas de la mejor manera posible y terminar". (Pedro, egresado de Ingeniería Química)

\section{Contexto de desarrollo de las estrategias: modalidad de cursado}

Esta categoría remitió al contexto curricular de cada carrera, que influenció en la adopción de ciertas estrategias para avanzar. Se observó que ciertas carreras proponen un cursado estructurado, que implica la organización de materias año a año y por cohorte, y los estudiantes pueden cursar todas las materias que les propone el plan. Otras carreras permiten un cursado no estructurado, en el que cursar materias se asocia a mecanismos de sorteo y orden de mérito, por lo que los estudiantes no tienen la posibilidad de cursar, de manera regular y sistemáti$\mathrm{ca}$, todas las materias que propone el plan, y se relaciona, con mayor frecuencia, con la posibilidad de rendir las materias libres y cursar de oyente.

\section{Estrategias para avanzar}

Esta categoría central refirió a las acciones y decisiones tomadas en función de las necesidades del cursado. La modalidad de cursado, estructurada o no estructurada, fue vertebradora en la organización en torno a la concurrencia a la facultad, al cursado de materias y al estudio. Es por ello que esta categoría varió en función de cada carrera, privilegió unas estrategias sobre otras e incorporó o desechó otras.

Avanzar en la carrera significó planificar acciones que favorecieran transitar por la propuesta curricular y aprobar materias, además de adoptar actitudes positivas y centralizar el tiempo en torno al estudio.

La Tabla 3 muestra las propiedades y dimensiones de la categoría estrategias para avanzar en función de la modalidad de cursado.

A modo ilustrativo de la primera propiedad de esta categoría, las estrategias de ajuste respecto de la fuente de aprendizaje y consulta de dudas, preponderante y diferenciada según modalidad de cursado, se muestran como la modalidad "pasilleo", la cual fue común en los egresados de la carrera de Abogacía (cursado no estructurado). Así es que el docente no fue reconocido en su función tutorial: 
Tabla 3. Propiedades de la categoría estrategias para avanzar, según la modalidad de cursado de la carrera

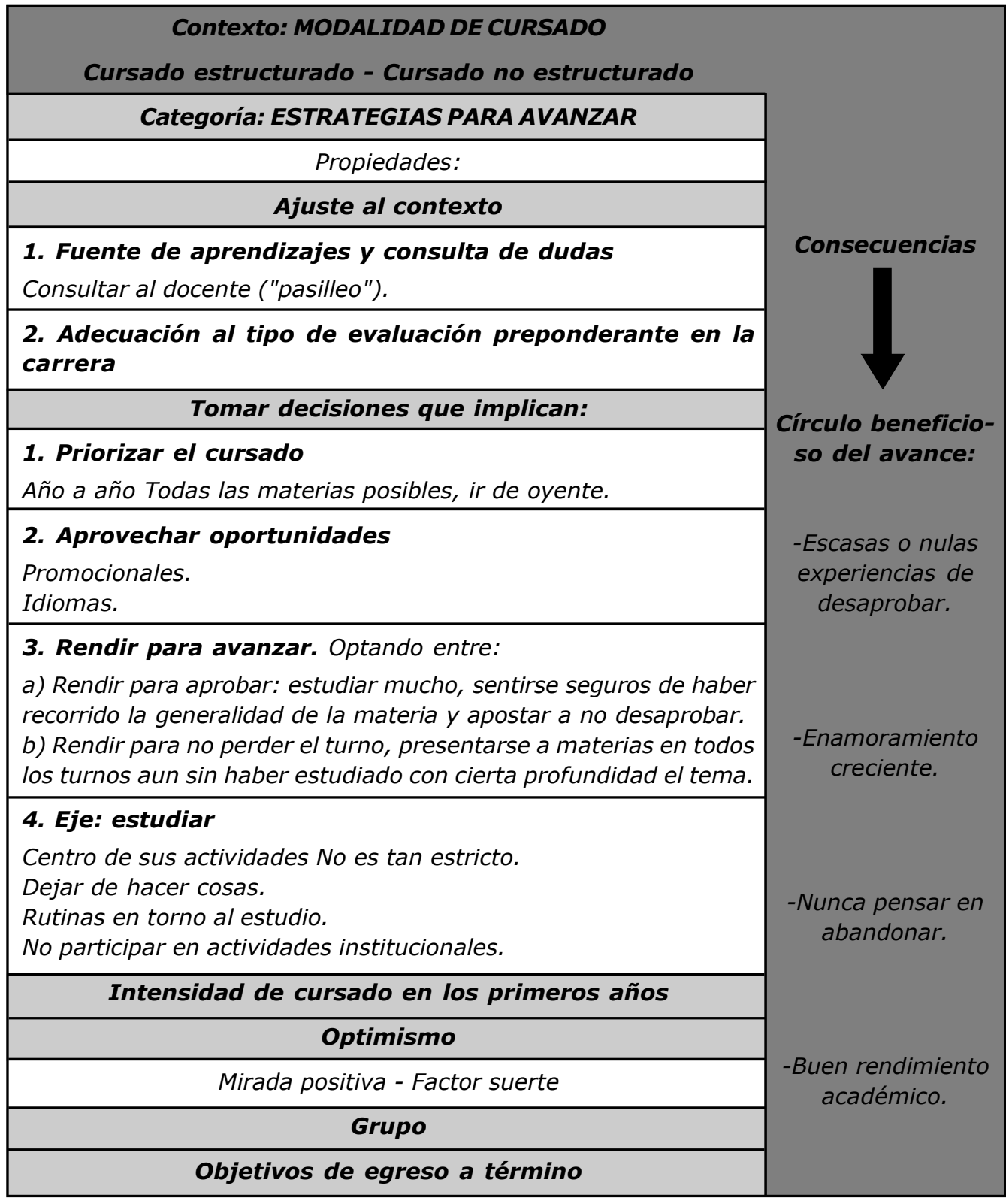


"Y compañeros, amigos, amigas que están estudiando. O la primera amiga mía que tuve, la hermana estudiaba Derecho, entonces siempre era nuestra referente [...] me guiaba por el pasilleo. [...] De hablar, de hablar con el resto... pasillo... Centro de estudiantes, sí, más o menos, porque en mi caso era la contra". (Carlos, egresado de Abogacía)

En cambio, en la modalidad estructurada, la fuente de consultas es el docente y los dispositivos que él construyó para cumplir con la función tutorial. Un dispositivo fue la clase de consultas, como lo expresó Andrés: "Y después las preguntas que teníamos todavía dudas, íbamos a los profesores y les preguntábamos" (Andrés, egresado de Ingeniería Química).

\section{La idea de trama desde la perspectiva de la complejidad}

El análisis de los datos permitió la obtención de categorías centrales que, conjuntamente con sus propiedades y dimensiones, dieron cuenta del tejido imbricado que sostiene la posibilidad de egresar a término en las carreras estudiadas. Es así que emerge la idea de la trama como metáfora para expresar las íntimas interrelaciones entre las categorías centrales y sus propiedades. Hablar de trama remite al paradigma de la complejidad, en el que se sitúa al ser humano, la naturaleza y las relaciones con ella, en un todo que se encuentra entrelazado (Morin, 2009).

Admite una visión de conjunto en la que no resalta la linealidad, sino la relacionalidad indisociable entre los elementos.

La trama no aparece inmediatamente visible, pero es lo que sostiene y caracteriza las trayectorias de cada uno de los entrevistados. En ella, podemos reconocer puntos de este tejido, a la vez que la lana con la cual se van tejiendo estos puntos forma un entrelazado irreductible a la simplicidad y a la causalidad.

Es así que un aporte de la investigación permitió tensionar la propuesta de la teoría fundamentada acerca de la necesidad de conformar una matriz que -claramente (simplemente)- muestre las ideas construidas en función de un fenómeno.

Strauss y Corbin (2002) presentan la idea de matriz en un corrimiento de la noción de causa y efecto, a la vez que proponen la consideración de las condiciones contextuales macro y micro como intervinientes y generadoras de consecuentes en el fenómeno estudiado. Los mencionados autores expresan, como una de las limitaciones del diagrama propuesto para graficar la matriz condicional, el hecho de aparentar cierta linealidad y proponen el uso de otras metáforas como las de "las bolas de billar" o "el caleidoscopio".

En esta investigación, las categorías pretenden comprender el fenómeno estudiado, a la vez que identifican elementos intervinientes y consecuentes, aunque es importante destacar que una categoría por sí misma no explica el fenómeno, ni siquiera la suma de ellas. Solo es explicado en términos del tejido entre ellas, sus propiedades y el contexto. La propuesta es distinguir sin desarticular. 


\section{Los puntos de la trama}

Sintéticamente, en relación a los hallazgos, se puede decir que la categoría equipaje se vinculó en un contínuum con la autopercepción estudiosa y ambas, de manera conjunta y entrelazada, condicionaron las estrategias para avanzar. Estas, a su vez, se comprenden en el contexto de la modalidad de cursado propia de cada carrera.

Con relación a la discusión teórica de las mencionadas categorías, se encontró que el equipaje se conformó en función del valor familiar otorgado al estudio como principal modo de ascenso social y un continuo monitoreo desde la escolaridad previa hasta la misma universidad. En este contexto, se constituyeron las buenas experiencias educativas previas, las que, a su vez, se instauraron en un contínuum dialéctico traducido en una autopercepción estudiosa. Aquí, el aporte de las teorías sociológicas de la educación, principalmente la denominada New Directions Sociology o Nueva Sociología de la Educación, permite echar luz respecto del rol central otorgado a la capacidad individual y a los antecedentes familiares como determinantes del éxito educativo y propone la existencia de un condicionante de clase social en la explicación del éxito o fracaso en los estudios. Sostiene que los alumnos provenientes de hogares de clase media tienen más probabilidad de éxito educativo porque, en su proceso de socialización, han desarrollado capacidades cognitivas y lingüísticas, valores, actitudes y aspiraciones que concuerdan con las exigencias de la educación formal (Bourdieu \& Passeron, 2009).

En este sentido, el apoyo económico generó la posibilidad de no trabajar y tener por eje el estudio, y, cuando este apoyo no fue posible, fue altamente compensado por el monitoreo familiar a través del apoyo emocional. Dicho esto, no puede dejar de mencionarse la estructura curricular que, en la mayoría de los casos, se presentó organizada para estudiantes sin otra obligación que la de estudiar. Este aspecto es estudiado por la sociología del currículum, que entiende a este como un instrumento de control social porque implica la imposición de significados y la distribución de conocimientos en referencia a las limitaciones que ciertos grupos encuentran para acceder a determinados ámbitos del saber (Young, 1971). Esto permite pensar en qué medida las universidades consideran la situación de estudiantes no tradicionales: primera generación familiar en la universidad, estudiantes de clase trabajadora o con dificultades socioeconómicas, adultos que trabajan, mujeres con cargas familiares, discapacitados, personas de origen inmigrante (González Monteagudo, 2010; Rama, 2007).

También, el mandato familiar de estudiar (más o menos explícito), el ser -o nola primera generación de estudiantes universitarios y el monitoreo familiar se hicieron presentes en los relatos de los entrevistados. Allí se destaca la incidencia favorable del medio familiar en el desempeño académico de los estudiantes cuando facilita el acceso directo al acervo cultural, a hábitos e instrumentos de trabajo intelectual.

En particular, el ser la primera generación de estudiantes universitarios es considerado, por diversas investigaciones (Ezcurra, 2013), como un factor condicionante adverso. 
Sin embargo, los casos de estudiantes cuyos padres no completaron los estudios primarios o secundarios permiten cuestionar la figura de "heredero" propuesta por Bourdieu y Passeron (2009). Aspecto consonante con datos aportados por los Anuarios de Estadísticas Universitarias del Ministerio de Educación de entre fines de la década de 1980 y 2006 que revelan, en primer lugar, un aumento de la matrícula en la década de los noventa $y$, en segundo lugar, que dicha matrícula estuvo conformada -principalmente- por estudiantes que trabajaban para sostener sus estudios y cuya procedencia social fue variable (Carli, 2012). Aunque el porcentaje de estudiantes que se gradúan se ubica ampliamente dentro de la población proveniente de hogares entre los quintiles más altos (García de Fanelli, 2014b).

La paradoja presentada anteriormente no deja más que resaltar la complejidad de la trama del egreso a término con relación a la imbricación indisociable entre las categorías que la conforman.

En el mismo sentido, la preparación académica preuniversitaria y la capacidad de financiamiento de los estudios son consideradas por Tinto (1987) variables previas y condicionantes de la integración de los estudiantes a la vida universitaria.

Con relación a lo expuesto y estableciendo cierta distinción, se señala que -en los casos estudiados- no se establecería un corte entre el bagaje previo a la universidad y el trayecto universitario, ya que los mensajes y actitudes de estímulo, sostén y supervisión por parte de la familia perduraron durante el transcurso de los estudios. Esto es lo que se denominó monitoreo familiar y se reconoció en un contínuum que pervivió durante la vida universitaria.

La generalidad de las investigaciones acerca del rol de la familia en la universidad se circunscribe al contexto socioeconómico y cultural que ofrece como capital previo al ingreso universitario. La familia, desde un lugar de "supervisión" de los estudios, no ha tenido un rol protagónico en la investigación actual.

Existen materiales que refieren al soporte familiar durante la transición a los estudios universitarios (primer año) como predictor de la persistencia universitaria, conjuntamente con la autoeficacia académica, la integración social y la tasa de estrés (Figuera, Dorio \& Forner, 2003). Así también, algunas universidades extranjeras han creado guías para familias que colaboran en la preparación para el ingreso a la universidad, especialmente en un contexto altamente competitivo, como es el ingreso a la universidad en países como los Estados Unidos (Universidad de Minnesota, 2014). Igualmente, no es un aspecto estudiado en profundidad.

Respecto de las buenas experiencias previas, el haber recibido algún tipo de reconocimiento formal durante las trayectorias educativas, tales como haber sido abanderado, escolta de bandera o participar del cuadro de honor, permitió articular la manera en que algunos ritos escolares contribuyen a la configuración de subjetividades. En este caso, subjetividades que no perciben el estudio como un obstáculo.

En este sentido, se sostiene que la educación es una práctica social productora de sujetos: "la escuela enseña diferencias, jerarquías, tabicamientos e incluso podemos pensar que 'en-seña', deja marca, rotula" (Vain, 2018, p. 16). 
Estas buenas experiencias educativas previas se vincularon con el carácter tenaz de la subjetividad. Podría sostenerse que estas experiencias positivas con respecto a las trayectorias educativas forjaron subjetividades con cierto grado de confianza en sí mismas como para plantearse objetivos y cumplirlos. Uno de estos objetivos es egresar a término.

En términos generales, la autopercepción estudiosa puede ser leída como las autodefiniciones (mediadas socialmente) que realizaron los sujetos participantes de la investigación con relación a sí mismos en el contexto del egreso a término.

Muchas investigaciones han estudiado diferentes constructos vinculados a cuestiones psicoemocionales de estudiantes universitarios, tales como el bienestar psicológico, la asertividad y el rendimiento académico (Velásquez et al., 2008); la vulnerabilidad al estrés, el apoyo social percibido, las habilidades comunicativas y la asertividad (Román Collazo \& Hernández Rodríguez, 2011); la inteligencia emocional (Martínez Olivera, González De La Fuente \& Mora Guevara, 2006); competencias socioemocionales (Castejón, Cantero \& Pérez, 2008). Asimismo, aquí se resalta la dimensión simbólica, es decir, los significados con los que los sujetos en interacción social construyen la realidad desde su propia perspectiva.

Es así que el autopercibirse como aplicado, tenaz y autoexigente es una construcción dinámica, pivote para la generación de estrategias para avanzar y ajustadas al contexto de la modalidad del cursado.

Estas estrategias podrían vincularse con lo que Carli (2012) denomina "tácticas de persistencia":

Aquellos [estudiantes] que llegaron al horizonte de la graduación, y que pueden calificarse de alguna manera de "exitosos", transitaron la vida universitaria implementando un conjunto de prácticas, acciones y reflexiones que les permitieron sortear dificultades, permanecer y apropiarse de la universidad. (p. 18)

En la definición de esta autora, es interesante la mención al carácter artesanal de dichas tácticas, lo que remite a la escasa mediación institucional en su diseño. Este aspecto coincide con una de las estrategias de avance hallada, la de ajuste, que señala como la principal fuente de aprendizajes y consulta al pasilleo, entendido como la búsqueda y obtención de información relevante a partir de la consulta con compañeros.

Es así que muchas de las estrategias utilizadas por los entrevistados supusieron la consecución del llamado oficio de estudiante (Coulon, 1995). Este proceso implica tres fases: el tiempo de extrañeza -no pertenencia-, en el curso donde el estudiante entra en un universo desconocido; el tiempo de aprendizaje, durante el cual se adapta progresivamente y, por último, el tiempo de afiliación, período en que se observa un relativo dominio que se manifiesta notoriamente por la capacidad de interpretar, incluso transgredir, las reglas.

Por lo tanto, el aprendizaje del oficio de alumno se logra, en definitiva, cuando se ha logrado la afiliación, es decir, cuando el estudiante "sabe identificar los códigos implícitos del trabajo intelectual, cuando oye lo que no se ha dicho y ve lo que 
no se ha identificado, cuando ha interiorizado lo que en un principio parecía externo a él mismo" (Coulon, 1995, p. 161).

Si bien los sujetos participantes de la investigación podrían ubicarse dentro de la fase de afiliación, dado que han finalizado exitosamente sus carreras, se pueden distinguir algunas de las estrategias que podrían haber colaborado en el pasaje del extrañamiento a la afiliación.

Los hallazgos muestran estrategias que podrían contener un tinte de mayor adaptación al contexto, como la estrategia de ajuste ya mencionada anteriormente, que incluye la selección de fuentes de aprendizaje y la adecuación al tipo de evaluación preponderante en la carrera. Como también estrategias que implican una posición más activa por parte de los estudiantes respecto a la toma de decisiones vinculadas a la priorización del cursado, el aprovechamiento de oportunidades, el rendir para avanzar y el tornar el estudio como eje en sus vidas.

Dichos aspectos pueden vincularse con el concepto de compromiso o implicación estudiantil (engagement) (Ezcurra, 2013), que remite al "tiempo en tarea", por lo tanto, a la dedicación al estudio. Dicho lo anterior, no puede dejar de señalarse la estrategia de intensificar el cursado durante los primeros años, una cuestión que -sustentada en la motivación- dio fuerza y empuje a este trayecto inicial en el que se hace necesario accionar en pos de salir de la fase de extrañamiento.

Con relación a lo expresado, existe un campo de investigación específico que dedica su estudio a los procesos de transición académica, especialmente en el marco anglosajón, donde constituye un área de estudio destacada (Figuera, Dorio \& Forner, 2003). Los estudios sostienen la relevancia de este periodo, ya que supone el primer balance de congruencia entre las opciones y elecciones del estudiante y la adecuación de sus proyectos y expectativas a la realidad, como también se señala el desenvolvimiento durante este periodo como predictor del éxito posterior (Porto, 2007; Giuliodori, Gertel, Casini \& González, 2010; Ezcurra, 2013).

Otra estrategia, también estudiada por la literatura académica, es la conformación de un grupo, que podría significar un modo compensador ante la falta de lazos institucionales. Con relación a este punto, Carli (2012) expone una hipótesis con respecto a la configuración de la sociabilidad en la vida universitaria, como conformada "en los límites de las instituciones, proveedoras de un soporte frente a la debilidad estratégica de las facultades como ámbitos cada vez menos sacralizados" (p. 167).

El grupo ancla al estudiante en la institución, crea un espacio de reconocimiento personal y representa la simbiosis de los componentes intelectuales y socioafectivos que se requieren para realizar la carrera. Es un espacio de socialización en el que se interiorizan las pautas académicas y profesionales.

Las características halladas (homogeneidad, amistad, apoyo y el estudio como eje) permiten visualizar la manera en que este ámbito social combina -en iguales proporciones- aspectos afectivos, identitarios y académicos.

Incluso, y ya de manera individual, esta centralidad del estudio resultó incompatible con el desarrollo de otras actividades propias de la vida estudiantil, como 
la participación en centros de estudiantes o cátedras. Este aspecto entra en contradicción con una de las medidas que propone Tinto (1989) con relación a la integración social del estudiante en la universidad (una de las dimensiones de la persistencia, junto con la integración académica), que es la participación en actividades extracurriculares.

Parecería que los estudiantes que egresaron a término encontraron, en el grupo, la canalización de la motivación social. Asimismo, esta estrategia puede vincularse con el tener objetivos de egreso a término, los cuales se conformaron en diferentes momentos de la carrera; en algunos casos, al inicio y -en otros- al medio o al final.

Otro aspecto interesante de resaltar dentro de las estrategias de avance es el optimismo como elemento interviniente a la hora de enfrentar situaciones estresantes, como los exámenes. La psicología positiva, que busca comprender los procesos que subyacen a las cualidades y emociones positivas del ser humano (Poseck, 2006), permite echar luz acerca de esta estrategia, que podría considerarse como un factor de protección o encuadrarse dentro de lo que podría entenderse como "emociones positivas". Investigaciones como la de Londoño Pérez (2009) demuestran que el optimismo es determinante, no sólo de la permanencia durante los estudios universitarios, sino que también predice el abandono y repetición de asignaturas, ambas consideradas señales de riesgo para la deserción.

Otro aspecto de los hallazgos tiene que ver con las consecuencias de las estrategias puestas en juego durante el cursado. En coherencia con el objetivo de dichas estrategias -lograr el avance en la carrera- y con una mirada que escapa a la linealidad en el fenómeno del egreso a término, se describió el círculo beneficioso del avance, que está conformado por las escasas o nulas experiencias de desaprobar, el enamoramiento creciente, el nunca pensar en abandonar y el buen rendimiento académico. Estos aspectos actuarían en retroalimentación permanente al fortalecer los puntos de la trama, ya que intervienen aspectos de motivación, persistencia y autoeficacia.

Con esto se pretende señalar la íntima relación entre rendir para avanzar (rendir para aprobar y rendir para no perder el turno), el tener escasas o nulas experiencias de desaprobar y el rendimiento académico.

A modo de cierre, se podría plantear la posibilidad de adaptación activa por parte de los estudiantes, que aprovecharon oportunidades y construyeron estrategias para avanzar según los plazos establecidos por el plan de estudios, lo que implica la alineación en términos del oficio de estudiante. (2002):

En este sentido, estos hallazgos coinciden con lo postulado por Baeza Correa

Los jóvenes construyen "saberes" y "saber hacer" que les permiten dar un "sentido" y "significado" propio a lo que realizan. Estos "saberes" y "saber hacer" lo adquieren en un proceso de apropiación que tiene como característica ser colectivo y situado. No conocer estos sentidos y significados propios, como a su vez las estrategias que desarrollan para posibilitar su 
protagonismo, pueden llevar a una explicación o relación no válida con ellos o a una elaboración de una política de atención inadecuada. (p. 163)

\section{A modo de conclusión}

Parte de las recomendaciones de la investigación, que fue sustento de este artículo, se vincula con la posibilidad, para las instituciones universitarias, de utilizar las categorías como modo de "diagnóstico e intervención" para las trayectorias académicas.

Es por esto que se expone una propuesta preliminar que requiere de ajustes y trabajo en terreno para ser probada y mejorada. Cabe clarificar que los aspectos y tópicos que se señalan no deben ser interpretados en una relación de predestinación; esto significaría pensar que la consideración de uno de los tópicos por sí mismo explicaría el abandono o el egreso. Bajo ningún aspecto se los valora como relaciones disociables o de causa y efecto. La mirada compleja debe estar presente para evitar cualquier intento de simplificación de la realidad (ver Tabla 4).

Reconocer los tópicos y los aspectos a tener en cuenta permitiría diseñar apoyos y dispositivos psicopedagógicos y académicos que coadyuven a un tránsito exitoso por la universidad para evitar -en algunos casos- dilaciones en los estudios que redunden en agotamiento, desgaste y abandonos posteriores.

Esto significa resaltar el valor que tienen y deberían asumir las instituciones educativas en las trayectorias de los estudiantes, más allá de sus equipajes y autopercepciones estudiosas, especialmente en lo que refiere a la construcción de estrategias de avance.

Ezcurra (2013) señala la manera en que las instituciones delegan toda la responsabilidad de la deserción y dilación a los mismos estudiantes. Por lo que los dispositivos generados asumirían "innovaciones periféricas", ya que el rol de las universidades es decisivo para el desempeño académico y la persistencia. Así que el lugar de la enseñanza cobra valor en la medida en que pueda generar efectos relevantes en el desempeño estudiantil.

Es por esto que las propuestas de Comunidades de Aprendizaje (Tinto, 1993), Seminarios de Primer Año (Mills, 2009), Tutoría de Pares (Sola Villazón \& De Pauw, 2004; Figueroa y Pasqualini, 2008), Alfabetización Académica (Carlino, 2005), entre otras, apuntan a enseñar lo implícito, tal como expone Ezcurra (2013), en especial para el primer año.

Como conclusión y a modo de apertura a nuevas indagaciones, y en tensión con la disciplina marco de la investigación, es que se acuerda con lo expresado por Lucarelli (2009), quien señala que la universidad, como objeto de estudio, en su complejidad, admite un abordaje polifacético y desde múltiples enfoques disciplinarios. Esta idea permite aventurar el pensamiento en dirección a lo que podría ser una psicopedagogía dedicada explícitamente al ámbito universitario, una psicopedagogía universitaria como campo específico de abordaje que requiere de la sistematización de trabajos en terreno, además de la elaboración teórica propia disciplinar. 
Tabla 4. Propuesta de intervención con base en categorías centrales y propiedades

\begin{tabular}{|c|c|}
\hline \multirow[t]{2}{*}{ Tópicos a observar } & \multirow[t]{2}{*}{ Aspectos a considerar } \\
\hline & \\
\hline Mandato familiar de estudiar & $\begin{array}{l}\text { Grado de expectativas familiares acerca de obtener } \\
\text { un título universitario. }\end{array}$ \\
\hline $\begin{array}{l}\text { Ser o no la primera generación } \\
\text { de estudiantes universitarios }\end{array}$ & $\begin{array}{l}\text { Existencia de experiencias de trayectorias universita- } \\
\text { rias en la familia. }\end{array}$ \\
\hline Monitoreo familiar & $\begin{array}{l}\text { Grado de supervisión o acompañamiento de la familia } \\
\text { acerca de los estudios. }\end{array}$ \\
\hline Apoyo económico familiar & $\begin{array}{l}\text { Sostenimiento económico. Estudiar sin trabajar o es- } \\
\text { tudiar y trabajar. }\end{array}$ \\
\hline $\begin{array}{l}\text { Buenas experiencias educati- } \\
\text { vas previas }\end{array}$ & $\begin{array}{l}\text { Valoración acerca de las experiencias educativas an- } \\
\text { teriores: bagaje de conocimientos para la universi- } \\
\text { dad, rendimiento escolar. }\end{array}$ \\
\hline \multicolumn{2}{|r|}{ Autopercepción estudiosa } \\
\hline Ser aplicado & $\begin{array}{l}\text { Percepción de sí mismo como dedicado al estudio, } \\
\text { responsable con esa actividad. }\end{array}$ \\
\hline Ser autoexigente & $\begin{array}{l}\text { Percepción de sí mismo como buscador de buenos y } \\
\text { mejores resultados. }\end{array}$ \\
\hline Ser tenaz & $\begin{array}{l}\text { Percepción de sí mismo como capaz de cumplir los } \\
\text { objetivos que se propone. }\end{array}$ \\
\hline \multicolumn{2}{|r|}{ Estrategias para avanzar } \\
\hline $\begin{array}{l}\text { Ajuste al contexto: } \\
\text { - Fuente de aprendizajes y con- } \\
\text { sulta de dudas } \\
\text { - Adecuación al tipo de evalua- } \\
\text { ción preponderante en la carrera }\end{array}$ & $\begin{array}{l}\text { Poder reconocer elementos del contexto académico } \\
\text { que permitan decidir dónde obtener mejores respues- } \\
\text { tas a sus consultas y dudas, como también discernir } \\
\text { las modalidades de evaluación preponderantes. }\end{array}$ \\
\hline $\begin{array}{l}\text { Tomar decisiones que implican: } \\
\text { - Priorizar el cursado } \\
\text { - Aprovechar oportunidades } \\
\text { - Rendir para avanzar } \\
\text { - Estudiar como eje }\end{array}$ & $\begin{array}{l}\text { Reconocer la posibilidad de decidir en torno a los tó- } \\
\text { picos presentados: reconocer el valor de asistir a } \\
\text { clases y el régimen de asistencia y promoción; plani- } \\
\text { ficar el avance a partir de rendir materias; otorgar } \\
\text { espacio y tiempo para el estudio. }\end{array}$ \\
\hline $\begin{array}{l}\text { Intensidad de cursado en los } \\
\text { primeros años }\end{array}$ & $\begin{array}{l}\text { Posibilidad de implicarse (engagement) en el estudio } \\
\text { al inicio de la carrera. }\end{array}$ \\
\hline Optimismo & $\begin{array}{l}\text { Grado de optimismo y presencia de factores protecto- } \\
\text { res. Tener la posibilidad de experimentar emociones } \\
\text { positivas, como también la inteligencia emocional. }\end{array}$ \\
\hline Grupo & $\begin{array}{l}\text { Posibilidad de entablar relaciones con pares que abo- } \\
\text { nen tanto los vínculos afectivos como el aprendizaje } \\
\text { colaborativo. }\end{array}$ \\
\hline Objetivos $a$ & $\begin{array}{l}\text { Grado de presencia de objetivos académicos (egreso, } \\
\text { avance, egreso a término). }\end{array}$ \\
\hline
\end{tabular}




\section{Referencias bibliográficas}

Argentina. Ministerio de Educación de la Nación (2005). Anuario de Estadísticas universitarias. Recuperado el 23 de marzo de 2016, de: https:// www.argentina.gob.ar/educacion/planeamiento/info-estadistica/anuarios.

Argentina. Ministerio de Educación de la Nación (2006). Anuario de Estadísticas universitarias. Recuperado el 30 de abril de 2016, de: https://www.argentina.gob.ar/ educacion/planeamiento/info-estadistica/anuarios.

Baeza Correa, J. (2002). Leer desde los alumnos(as), condición necesaria para una convivencia escolar democrática. Educación secundaria. Un camino para el desarrollo humano. Santiago de Chile: UNESCO/OREALC.

Barsky, O., Sigal, V. \& Dávila, M. (2004) (Coords.). Los desafíos de la Universidad Argentina. Buenos Aires: Siglo Veintiuno.

Bourdieu, P. \& Passeron, J. C. (2009). Los herederos. Los estudiantes y la cultura. Buenos Aires: Siglo Veintiuno.

Brunner, J. J. \& Ferrada Hurtado, R. (2011) (Eds.). Educación Superior en Iberoamérica. Informe 2011. Santiago de Chile: CINDA-UNIVERSIA.

Cabrera, L., Tomás, J., Álvarez, P. \& González, M. (2006). El problema del abandono de los estudios universitarios. RELIEVE, 12(2), 171-203. Recuperado el 12 de junio de 2015 de: http://www.uv.es/RELIEVE/v12n2/RELIEVEv12n2_1.htm.

Camarena, R., Chávez, A. M. \& Gómez, J. (1985). Reflexiones en torno al rendimiento escolar y a la eficiencia terminal. Revista de la Educación Superior, 14(53), 1-17. Recuperado 23 de abril de 2016 de: http://publicaciones.anuies.mx/revista/53.

Carli, S. (2012). El estudiante universitario. Hacia una historia del presente de la educación pública. Buenos Aires: Siglo Veintiuno.

Carlino, P. (2005). Escribir, leer y aprender en la universidad. Una introducción a la alfabetización académica. Buenos Aires: Fondo de Cultura Económica.

Castaño, E., Gallón, S., Gómez, K. \& Vásquez, J. (2006). Análisis de los factores asociados a la deserción y graduación estudiantil universitaria. Lecturas de Economía, 65, 9-36.

Castejón, J. L., Cantero, M. P. \& Pérez, N. (2008). Diferencias en el perfil de competencias socio-emocionales en estudiantes universitarios de diferentes ámbitos científicos. Revista Electrónica de Investigación Psicoeducativa, 6(15), 339-362. Recuperado el 12 de mayo de 2015, de: http://www.investigacionpsicopedagogica.org/revista/new/ContadorArticulo.php?267.

Coulon, A. (1995). Etnometodología y educación. Barcelona: Paidós.

Cuéllar Saavedra, O. \& Bolívar Espinoza, A. (2006). ¿Cómo estimar la eficiencia terminal en la Educación Superior? Notas sobre su estatuto teórico. Revista de la Educación Superior. ANUIS, 35(139), 7-23. 
Edel Navarro, R. (2004). Educación a distancia y eficiencia terminal exitosa: el caso de la Sede Tejupilco en la Universidad Virtual del Tecnológico de Monterrey. RED, Revista de Educación a Distancia, 3(12), 1-23.

Ezcurra, A. M. (2013). Igualdad en la educación superior. Un desafío mundial. Buenos Aires: Universidad Nacional de General Sarmiento.

Ezcurra, A. M. (2014, 15 de junio). La "inclusión excluyente": más alumnos y más deserción. Perfil. Recuperado el 11 de junio de 2016 de: http://www.perfil.com/ sociedad/La-inclusion-excluyente-mas-alumnos-y-mas-desercion-201406150044.html.

Figuera, P., Dorio, I. \& Forner, A. (2003). Las competencias académicas previas y el apoyo familiar en la transición a la universidad. Revista de Investigación Educativa, 21(2), 349-36.

Figueroa, P. \& Pasqualini, V. (2008). Tutorías de pares: un lugar común que posibilita la experiencia de habitar la universidad como sujeto epistémico-político. Anales del 30 encuentro Nacional sobre Ingreso Universitario. Políticas, prácticas y saberes sobre el Ingreso a las Carreras de Humanidades, Ciencias Sociales y Artes en las Universidades Públicas [CD]. Río Cuarto, Córdoba.

García de Fanelli, A. M. (2005). Acceso, abandono y graduación en la educación superior argentina. En Sistema de Información de Tendencias Educativas en América Latina (SITEAL), Educación superior. Acceso, permanencia y perfil social de los graduados comparados con los egresados de la educación media (pp. 2-17). Buenos Aires: OEI.

García de Fanelli, A. (2014a). Rendimiento académico y abandono universitario: Modelos, resultados y alcances de la producción académica en la Argentina. Revista Argentina de Educación Superior, 6(8), 9-38.

García de Fanelli, A. (2014b). Inclusión social en la Educación Superior Argentina: indicadores y políticas en torno al acceso y a la graduación. Páginas de Educación, $7(2), 275-297$.

García de Fanelli, A. (2015). La cuestión de la graduación en las universidades nacionales de la Argentina: Indicadores y políticas públicas a comienzos del siglo XXI. Propuesta Educativa, 1(24), 17-31.

Giuliodori, R., Gertel, H., Casini, R. \& González, M. (2010). El desempeño de los estudiantes de una universidad masiva en Argentina, su relación con la historia académica previa en el nivel secundario y con factores socio-económicos. En J. M. Roig Cotanda \& L. E. Vila Lladosa (Coords.), Investigaciones de Economía de la Educación (pp. 317-326). Valencia: Fundación Universidad-Empresa.

Glaser, B. \& Strauss, A. L. (1967). The Discovery of Grounded Theory. Strategies for Qualitative Research. Chicago: Aldine.

González Monteagudo, J. (2010). Biografía, identidad y aprendizaje en estudiantes universitarios no tradicionales. Estudio de caso de una mujer trabajadora. Profesorado. Revista de Currículum y Formación de Profesorado, 14(3). Recuperado el 8 de julio de 2016, de: http://www.ugr.es/ recfpro/rev143ART9.pdf. 
Landi, J. \& Giulodori, R. (2001). Graduación y deserción en las universidades nacionales. En A. Jozami \& E. Sánchez Martínez (Comps.), Estudiantes y profesionales en la Argentina: una mirada desde la Encuesta Permanente de Hogares (pp. 79-103). Buenos Aires: Editorial de la Universidad Nacional de Tres de Febrero.

Londoño Pérez, C. (2009). Optimismo y salud positiva como predictores de la adaptación a la vida universitaria. Acta colombiana de psicología, 12(1),95-107.

López Suárez, A., Albíter Rodríguez, A. \& Ramírez Revueltas, L. (2008). Eficiencia terminal en la educación superior, la necesidad de un nuevo paradigma. Revista de la Educación Superior, 37(146), 135-151.

Lucarelli, E. (2009). Teoría y práctica en la universidad. La innovación en las aulas. Buenos Aires: Miño y Dávila.

Martínez Olivera, A. L., González De La Fuente, M. V. \& Mora Guevara, J. L. A. (2006). Factores vinculados con el éxito académico en estudiantes universitarios. Universidad Nacional Autónoma de México. Recuperado el 8 de agosto de 2013, de: papyt.xoc.uam.mx/media/bhem/docs/pdf/21.PDF.

Mills Reid, K. (2009). A multiple case study of college First Year Seminars. Las Vegas: University of Nevada. Recuperado el 21 de marzo de 2016, de: https:// pdfs.semanticscholar.org/cdfd/8556fccb9ea4a694b8f477f5854eefb1fa38.pdf? _ga $=2.27544060 .1920665620 .1565227936-755840767.1565227936$.

Morin, E. (2009). Introducción al pensamiento complejo. Barcelona: Gedisa.

Oddino, S. (2003). La deserción estudiantil en las universidades nacionales argentinas: aproximación al problema con especial referencia a la Facultad Regional Villa María de la UTN. Tesis de Maestría, Universidad Nacional del Comahue.

Pérez Gonzáles, J. A. (2006). La eficiencia terminal en programas de licenciatura y su relación con la calidad educativa. Revista Electrónica Iberoamericana sobre Calidad, Eficacia y Cambio en Educación, 4(1), 130-148.

Pérez Lindo, A. (Coord.) (2007). Prospectiva de la Educación Superior Argentina 2020. Secretaría de Ciencia y Tecnología. Plan Estratégico Nacional de CTI Bicentenario (2006-2010). Ejercicio 2020: Escenarios y estrategias del CTI. Grupo Educación Superior. Trabajo preparado para la Comisión de Gestión de Recursos Humanos de la SECYT. Recuperado el 21 de marzo de 2014, de: http:// rapes.unsl.edu.ar/Publicaciones-Investigacion-Estudios-Educacion.

Porto, A. (2007). Mecanismos de admisión y rendimiento académico de los estudiantes universitarios. En A. Porto (ed.), Mecanismos de admisión y rendimiento académico de los estudiantes universitarios. Estudio comparativo para estudiantes de Ciencias Económicas (pp. 7-18). La Plata: Editorial de la Universidad de La Plata.

Poseck, B. V. (2006). Psicología positiva: una nueva forma de entender la psicología. Papeles del Psicólogo, 27(1), 3-8.

Rama, C. (2007). Los nuevos estudiantes en circuitos diferenciados de educación. En Unión de Universidades de América Latina y el Caribe, Educación Superior en 
América Latina y el Caribe: sus estudiantes hoy (pp. 13-42). México: Idea latinoamericana.

Román Collazo, C. A. \& Hernández Rodríguez, Y. (2011). El estrés académico: una revisión crítica del concepto desde las ciencias de la educación. Revista Electrónica de Psicología Iztacala, 14(2), 1-13.

Sánchez, F., Quirós, M., Reverón, C. \& Rodríguez, A. (2002). Equidad social en el acceso y permanencia en la universidad pública. Determinantes y factores asociados [Versión electrónica]. Centro de Estudios para el Desarrollo Económico (CEDE) de la Universidad de los Andes. Recuperado el 01 de marzo de 2015, de: http:// www.economia.uniandes.edu.co/content/download/.../D2002-16.PDF.

Sautu, R. (2005). Todo es teoría. Objetivos y métodos de investigación. Buenos Aires: Lumiere.

Sola Villazón, A. \& De Pauw, C. (2004). La tutoría de pares: una experiencia de lectura dialógica. Primer congreso Internacional Educación, Lenguaje y Sociedad. Universidad Nacional de La Pampa. Recuperado el 23 de abril de 2016, de: http:/ /miaprendizaje.uchile.cl/lea/tutores/wp-content/uploads/Sola-A.-de-Pauw-C.-2004.La-tutor\%C3\%ADa-de-pares-una-experiencia-de-lectura-dial\%C3\%B3gica.pdf.

Strauss, A. L. \& Corbin, J. (2002). Bases de la investigación cualitativa. Técnicas y procedimientos para desarrollar la teoría fundamentada. Antioquía: Universidad de Antioquía.

Tinto, V. (1987). Leaving College. Chicago: The University of Chicago Press.

Tinto, V. (1989). Definir la deserción: una cuestión de perspectivas. Revista de Educación Superior. Distrito Federal, México, Asociación Nacional de Universidades e Instituciones de Educación Superior (ANUIES), XVIII(3). Recuperado el 25 de marzo de 2015, de: http://publicaciones.anuies.mx/pdfs/revista/ Revista71_S1A3ES.pdf.

Tinto, V. (1993). Reflexiones sobre el abandono de los estudios superiores. Perfiles Educativos, 18(3) 35-52.

Universidad de Minnesota (2014). Guía para padres de familia de preparación para ir a la universidad y de carreras. Guía para apoyar los sueños de sus hijos. College Readiness Consortium.

Vain, P. D. (2018). Los rituales escolares y las prácticas educativas. Posadas: Editorial Universitaria de la Universidad Nacional de Misiones.

Velásquez, C., Montgomery, U. W., Montero, L. V., Pomalaya, V. R., Dioses, Ch. A., Velásquez, C. N., Araki, O. R. \& Reynoso, E. D. (2008). Bienestar psicológico, asertividad y rendimiento académico en estudiantes universitarios sanmarquinos. Revista IIPSI, Facultad de Psicología, UNMSM, 11(2), 139 - 152.

Young, M. (Comp.) (1971). Knowledge and Control. Londres: Collier-Macmillan. 